\section{Cahiers de Narratologie}

Analyse et théorie narratives

$7 \mid 1996$

Mélanges espace \& temps

\title{
Le roman pastoral à l'épreuve de l'histoire, étude narratologique de l'espace dans El Mirtilo o los pastores trashumantes de Pedro Montengón
}

Marc Marti

\section{CpenEdition}

Journals

Édition électronique

URL : https://journals.openedition.org/narratologie/11778

DOI : 10.4000/narratologie. 11778

ISSN : 1765-307X

Éditeur

LIRCES

Édition imprimée

Date de publication : 1 janvier 1996

Pagination : 49-62

ISSN : 0993-8516

Référence électronique

Marc Marti, « Le roman pastoral à l'épreuve de l'histoire, étude narratologique de l'espace dans El Mirtilo o los pastores trashumantes de Pedro Montengón ", Cahiers de Narratologie [En ligne], 7 | 1996, mis en ligne le 27 avril 2021, consulté le 05 mai 2021. URL : http://journals.openedition.org/ narratologie/11778; DOI : https://doi.org/10.4000/narratologie.11778

Ce document a été généré automatiquement le 5 mai 2021.

Article L.111-1 du Code de la propriété intellectuelle. 


\title{
Le roman pastoral à l'épreuve de l'histoire, étude narratologique de l'espace dans El Mirtilo o los pastores trashumantes de Pedro Montengón
}

\author{
Marc Marti
}

«Le roman est donc, dès le commencement, pétri dans une autre pâte que celle des genres achevés. Il est d'une nature différente. Avec lui, en lui, est né, dans une certaine mesure, l'avenir de toute la littérature. C'est pourquoi, une fois né, il ne pouvait simplement devenir un genre parmi les genres, ni établir avec eux les relations mutuelles d'une coexistence pacifique et harmonieuse. Face au roman, tous les genres commencent à résonner autrement. C'est un long conflit qui commence, pour « romaniser » les autres genres, pour les attirer dans la zone de contact de l'actualité inachevée ${ }^{1} »$. Mikhaïl Bakhtine

\section{INTRODUCTION}

\section{L'œuvre : contexte et argument}

1 Le roman. El Mirtilo o los pastores transhumantes de Pedro Montengón constitue d'abord une curiosité dans l'histoire de la littérature espagnole. Terminé en 1786, il n'a été publié qu'en 1795 par l'imprimeur madrilène Sancha (édition unique à ce jour). Ce roman est une curiosité non parce que le manuscrit a mis neuf ans à se transformer en livre - pour des raisons de procédure dans la censure et non pour son contenu - mais parce qu'il s'agit d'un roman pastoral. En effet, ce type d'ouvrage a connu une gloire éphémère - en gros presque un siècle, de 1550 à 1633 -, puisque d'après Avalle Arce, le dernier roman pastoral publié en Espagne date de $1633^{2}$. Mais, bien que cette œuvre soit isolée au niveau générique, il en va tout autrement quant à sa thématique ${ }^{3}$. Si au 
$\mathrm{XVIII}^{\mathrm{e}}$ on n'écrit plus de romans pastoraux, quelques textes classiques sont cependant réédités : Diana enamorada de Gil Polo, Arcadia de Lope de Vega, El pastor de Fílida de Gálvez Montalvo ${ }^{4}$. Il est aussi possible que Montengón, jésuite exilé qui vivait en Italie ait été plus sensible que les écrivains de la péninsule aux œuvres pastorales du Français Florian et du Suisse Gessner ${ }^{5}$.

2 L'argument de l'œuvre reprend la transhumance évoquée par le titre. Mirtilo, jeune poète dont l'intention était d'accéder à la célébrité en s'installant à la Corte, en repart très déçu et décide de devenir berger en Andalousie. En chemin, il rencontre un troupeau en transhumance qui se dirige vers les pâturages d'hiver d'Extrémadure. Il se joint au groupe de transhumants et se lie d'amitié avec Silviano, qui est aussi devenu berger pour fuir la ville. Le voyage est entrecoupé de duels poétiques (églogues, chansons, romances, etc.) lors des étapes de repos. Différents événements, comme des bals, un crime par jalousie ou une rencontre avec un loup se succèdent tout au long du récit. Un des épisodes les plus marquants est l'apparition d'un magicien, qui prédit que dans un futur proche, les transhumances disparaîtront et que la campagne se couvrira de champs de blé. Arrivé en Extrémadure, Mirtilo part à la recherche d'une terre pour s'installer. Après avoir péniblement escaladé une montagne escarpée, il découvre une vallée florissante où vivent deux femmes, une mère et sa fille. Cette dernière, qui n'a jamais vu d'homme de sa vie, se distingue par sa pureté et son innocence. Mirtilo en tombe amoureux. Le roman s'achève lorsque la mère décide de raconter au héros les péripéties de leur vie.

\section{Problématique et méthodologie}

3 Ce roman de Montengón, qui aurait plus de cent cinquante ans de retard au regard des classifications des historiens de la littérature nous intéresse à plusieurs titres. D'abord, il conviendrait de s'interroger les rapports (thématiques et formels) qu'entretient cette œuvre « résurgente » vis-à-vis de la tradition pastorale : imitation ou exercice de style, adaptation, parodie? Ensuite, il faut replacer l'œuvre dans son contexte, pourquoi écrire un roman pastoral à la fin du XVIII siècle ? Il nous semble que pour répondre à ces questions, une étude de l'espace pastoral serait la plus appropriée.

4 L'analyse partira de l'idée "qu'il existe un moyen terme [entre la notion d'autoréférentialité et celle de reflet du réel] pour l'analyse des textes romanesques, qui consiste à considérer que tous les romans renvoient, d'une certaine façon et à des degrés différents, à une réalité pré-construite et posée hors du langage ${ }^{6}$ » Afin d'aborder l'espace comme une catégorie textuelle à part entière, nous adopterons les propositions méthodologiques faites par Jacques Soubeyroux en distinguant trois niveaux d'analyse et de lecture de l'espace.

- une topographie mimétique : cette prise en compte du degré de mimétisme sera pour nous d'une importance capitale, en particulier dans le rapport littérature pastorale et réalité ;

- une toposémie fonctionnelle, orientée vers trois directions; l'étude sémiologique du lieu, qui étudiera le rapport entre lieux, personnages et actions, la caractérisation sociale et/ou symbolique des lieux, le fonctionnement diégétique de l'espace.

- le « symbolisme idéologique ", c'est-à-dire que l'on regroupera, ou on mettra en lumière les figurations symboliques de l'espace qui s'investissent dans le texte, et on montrera les implications idéologiques qu'elles ont presque toujours, car les symboles et les mythes ne 
sont pas un simple jeu culturel ou un simple procédé d'écriture : ils sont porteurs, parfois même les vecteurs privilégiés, du message idéologique ».

\section{TOPOGRAPHIE MIMÉTIQUE EL MIRTILO O LOS PASTORES TRASHUMANTES DE PEDRO MONTENGÓN}

L'action du roman est largement entrecoupée de pièces en vers. Nous avons, en théorie, la coexistence de deux espaces : un espace diégétique, où se déroule l'action; un espace extradiégétique, construit par les poésies. Ils ne sont cependant pas foncièrement différents car la plupart du temps l'un comme l'autre ne sont que des avatars du locus amœnus traditionnel avec cependant des fonctions distinctes, comme nous le verrons? ${ }^{7}$.

Le voyage des bergers est une succession d'étapes dans les lugares amenos qu'offre la nature :

«Montano voulut faire halte dans une combe, assez touffue, au milieu de laquelle serpentait lentement un doux ruisseau parmi les herbes hautes, où le bétail pouvait paître et s'abreuver à son aise. Ils se retirèrent à l'ombre de trois grands chêneslièges, où Montano ne voulut point que les rebecs de ses bergers restassent oisifs ${ }^{8}$.

Certaines étapes de cette transhumance ont lieu dans des bergeries, parfois à l'abord des villages ou des fermes: canadas (chemins de transhumance), majadas (bergeries), cabaña (cabane, troupeau), vecina aldea (le village voisin), el zaguán (le vestibule), la alquería (la ferme) 9 .

Le relief est évoqué dans une succession de vallées et de collines, paysage typique de la transhumance : valles (vallées), collados, lomas (collines), oteros (buttes), pendio, las laderas de los collados (les flancs des collines), pequeña cuesta (petite côte), barranco (combe) etc. On trouve cependant un relief plus escarpé à la fin du roman dont la fonction est de protéger la vallée merveilleuse découverte par le héros : peñas (montagnes/rochers), altas peñas (de hautes montagnes), cerros muy escarpados (des hauteurs abruptes), erizados riscos (des pics escarpés), aquellas serrezuelas (ces petites montagnes), larga cordillera (longue chaîne de montagnes), aquella cadena de altas peñas (cette chaîne de hautes montagnes).

9 Bien que le récit se déroule entièrement à la campagne, la ville est évoquée trois fois. La capitale que Mirtilo abandonne en début de roman: la corte, (la capitale), sa ville d'origine. Villa del reino de Valencia, enfin, les ruines romaines de la ville de Mérida : la ciudad de Mérida, con los monumentos de los romanos que allí se conservaban (la ville de Mérida et les monuments romains que l'on y conservait); esas ruinas (ces ruines), esos mármoles rotos (ces marbres brisés), mole destruida (amas de ruines), excelsos coliseos (éminents colisées), estas deformes piedras (ces pierres défigurées), paredes de templos destruidos (murs de temples détruits), desechos teatros y termas (théâtres et thermes en ruine).

10 Finalement, la topographie diégétique du Mirtilo fait avant tout référence à la littérature par sa succession de lugares amenos et non à la réalité. Le texte se distingue donc par une tendance assez marquée à l'autoréférentialité, c'est-à-dire un espace construit à partir d'éléments empruntés à la littérature. Cependant la brièveté des descriptions dans le corps de la narration témoigne d'une volonté de vraisemblance romanesque, qui se traduit aussi par le désir de faire coïncider le «réel» de la transhumance, structurant le déroulement temporel de l'œuvre, avec la série de clichés 
littéraires (locus amœenus), qui prennent ici des significations nouvelles. C'est en quelque sorte le chronotope classique de la pastorale que Montengón réutilise en le «romanisant», introduisant un temps linéaire (la transhumance) et non cyclique ${ }^{10}$. Cette volonté de réalisme aboutit finalement à une topographie qui superpose deux espaces: l'un auto-référentiel et littéraire: le cliché du locus amœnus et l'autre mimétique : l'Espagne du XVIII ${ }^{e}$ et son paysage de transhumance des régions du sud. Cependant, avant de tirer des conclusions définitives sur cette double référentialité, il convient de s'interroger sur les fonctions de l'espace dans le roman.

\section{TOPOSÉMIE FONCTIONNELLE}

\subsection{L'étude sémiologique du lieu}

11 Le locus amœnus domine donc le corps de la narration et il convient ici de déterminer quels sont ses caractéristiques dans le roman.

Ce topos réunit toujours au minimum les trois éléments essentiels de la tradition: l'ombre, la fertilité, l'eau, représentés par l'arbre, la prairie, le ruisseau ou la source ${ }^{11}$. L'ombre est évoquée en elle-même par les adjectifs ou les substantifs comme frondosidad, frondoso, frondosísimo (touffu, très touffu); par un ensemble d'éléments génériques comme árbol (arbre), bosque (bois), bosquecillo (bosquet), montes (forêt), matorrales (taillis) ou enfin par des arbres précis: sombría encina (yeuse ombreuse), acebuches (oliviers sauvages), tres grandes alcornoques (trois grands chênes-lièges), fresnos (frênes), arbustos (arbustes), sauces (saules).

14 La fertilité se rapporte surtout aux pâturages : crecida yerba (hautes herbes); dehesa fértil (prairie fertile); abundante pasto (gras pâturage); rico y fértil suelo (terre riche et fertile); viciosas matas (des mattes d'herbe touffues), frutales, árboles fructiferos (arbres fruitiers). L'eau, la plupart du temps un ruisseau ou une fontaine : claro arroyo (clair ruisseau); fuente (fontaine) : manso arroyuelo (doux petit ruisseau); un riachuelo (une petite rivière); el remanso cristalino (une eau calme et cristalline).

15 Le corps de la narration est assez pauvre en adjectifs et trois des quatre éléments subalternes du locus amœnus - le chant des oiseaux, les fleurs, la brise, les fruits - en sont absents. Seules les fleurs sont présentes et uniquement à la fin du roman, dans la vallée d'hivernage de Montano où apparaissent des lauriers roses en fleur ("floridas adelfas") et dans la vallée merveilleuse que découvre Mirtilo, des violettes et des giroflées ("violas y alelíes"). Il y a là une invraisemblance, puisque nous nous trouvons théoriquement vers la fin de l'automne (début de l'hivernage) où aucune de ces fleurs ne devraient apparaitre : le locus amœnus reprend en fait ses droits sur le roman et ses références sont dans la littérature et non dans la réalité. Les trois autres composantes du locus amœenus n'apparaissent que dans les chansons intercalées. Le chant des oiseaux :

«Dans les bois déjà touffus

Résonne le chant des oiseaux,

Chant d'amour.

Doux gazouillement ${ }^{12 "}$.

16 Les fleurs: flores (fleurs); florida alfombra (tapis de fleurs); valles floridos (vallées fleuries); tomillos (thym); romeros (romarin); nativas flores (fleurs en bouton); prado florido (pré fleuri). 
La brise : céfiros (zéphirs).

Les fruits : racimosa parra (la vigne vierge).

Tout au long du récit, l'utilisation du topos est explicite. Dans le corps de la narration, tout comme dans les chansons, le lieu décrit ou bien un de ses éléments constitutifs est souvent qualifié par l'épithète ameno ou delicioso : ameno valle (charmante vallée), ameno vallecito (charmante petite vallée), ameno bosque (délicieux bois), ameno sitio (endroit charmant), ameno y fértil trono de natura (charmant et fertile trône de la nature), amena y encantadora escena (scène charmante et enchanteresse), sitio delicioso (endroit délicieux), deliciosa fuente (délicieuse fontaine), delicioso prado (pré délicieux), deliciosos valles (délicieuses vallées), un lugar sombrío y delicioso (un endroit délicieux et ombragé). Cette qualification est parfois complétée par la solitude ou le silence : esta soledad (cette solitude mais aussi cet endroit solitaire), silenciosos fresnos (frênes silencieux), solitaria peña (montagne solitaire), solitarios cerros (colline solitaire), ameno desierto (charmant désert). Finalement, l'espace rural et pastoral est toujours valorisée.

Au contraire, la ville, espace extradiégétique, évoquée par les personnages, se charge de traits négatifs. La capitale est qualifiée de aquella Babilonia (cette Babylone), escollo de la fortuna (cet écueil de la fortune), ruidoso emporio de la fortuna y de la grandeza (bruyant marché de la fortune et de la grandeur), ciudadana turbulencia (effervescence citadine). Les ruines romaines de Mérida sont prétexte à une réflexion sur la relativité de la gloire, de l'ambition et de la puissance du temps qui passe ${ }^{13}$. Enfin, la ville est évoquée dans le thème du Menosprecio de corte y alabanza de aldea ${ }^{14}$, qui reprend les motifs suivants :

- Éloge de la pauvreté :

«[...] Moi. dans mon apparente pauvreté

Je suis heureux, car rien

Dans mon état ne m'inquiète

Ni peut inquiéter mon âme tranquille,

Car aux fastes des villes

Je préfère ces solitudes ${ }^{15}$ ».

- Mépris du luxe, de la richesse et des honneurs :

«Ici. parmi les bergers,

Tu connaîtras sans aucun doute,

Un bonheur plus pur; et d'autres satisfactions,

Exemptes des peines,

Qui viennent toujours troubler

Celles de ceux qui n'en connaissent

Que lorsqu'ils ont la chance

De bénéficier d'excellents emplois et de richesses

Car on dit qu'il y a là-bas bien peu

D'âmes satisfaites [...]

Ils peuvent bien imprégner

Leurs cheveux de parfums rares.

Et traîner de la soie tissée d'or [...]

Le riche n'a pas plus de soulagements que le pauvre ${ }^{16} \%$.

- Éloge du repos et de la liberté campagnarde :

«Mon père m'a toujours appris

A avoir les villes en horreur [....]

Le luxe qui y domine.

Ainsi que toutes ses hautes vanités.

Il encensait au contraire la douceur

De la paix de la vie campagnarde 
Et sa précieuse liberté, exempte

De lien servile ${ }^{17}$ »

21

Dans ce roman, l'unique fonction de la ville est de mettre en valeur les vertus de la campagne.

En plus de sa valeur esthétique et littéraire, le locus amœenus sert aussi la vraisemblance du récit romanesque. Il justifie l'étape, puisque le bétail trouve de quoi se nourrir et s'abreuver et il permet aux hommes de se reposer à l'ombre, de chanter et de se restaurer ;

«Le jour suivant, ils firent halte dans une vallée très fertile et touffue, où le bétail pouvait paitre librement, et eux se reposer à l'ombre de quelques oliviers sauvages, à côté d'une fontaine qui tombait d'une hauteur voisine dans un grand murmure. Là, pendant que les deux jeunes bergers qui accompagnaient Montano préparaient le repas, Montano demanda à Silviano et à Mirtilo de chanter une chanson ${ }^{18}$ ».

Au niveau esthétique, cette fonction du locus amœnus est une nouveauté. Auparavant, dans la littérature, il n'avait qu'une fonction décorative, alors qu'ici, il sert aussi la vraisemblance. Finalement donc, l'espace semble glisser du réalisme discursif (imitation des topoï traditionnels) au réalisme mimétique (imitation de l'espace réel) ${ }^{19}$.

\subsection{La caractérisation sociale et/ou symbolique du lieu}

Le Mirtilo, par sa nature et ses nombreuses références littéraires, fait partie des romans où l'espace doit aussi être envisagé dans sa dimension symbolique. Ce roman valorise particulièrement ce que Gilbert Durand appelle «l'imaginaire nocturne ${ }^{20 "}$.

Nous avons mis en valeur la présence de thèmes affectifs qui oppose l'espace de la transhumance à la ville: thématique du repos et de la liberté, éloge de l'humilité et condamnation de la richesse. La ville, repoussoir de la campagne, se charge d'attributs infernaux, ceux d'un univers hostile au repos et à la profondeur : le superficiel, la vaine ostentation; le vertige dangereux du luxe et de la richesse ; l'aveuglement des citadins face aux apparences ; la condamnation de l'ascension sociale, recherche des honneurs et des hautes charges auxquelles Mirtilo aspirait au début du roman et auxquelles il renonce pour vivre avec les bergers et finalement découvrir une vallée merveilleuse, qui a beaucoup à voir avec le mythe de l'âge d'or et du paradis terrestre.

Celle-ci réunit en effet tous les éléments du paradis de l'imaginaire nocturne. Intimité et «douce coalescence de l'homme et de son environnement ${ }^{21}$ ", que l'on trouve au début de la chanson que l'endroit inspire au héros:

«Paradis terrestre,

Dont la beauté nouvelle m'exalte

Et me transporte l'âme ${ }^{22 " ~}$

Dans ce paradis, l'image de la fertilité domine, avec en particulier l'arbre fruitier, synthèse de l'élément masculin et féminin (la terre et le bâton bourgeonnant) :

"Où que Mirtilo posât son regard, lui apparaissaient de multiples bosquets, disposés en un délicieux désordre, quelques-uns étaient composés d'arbres fruitiers, à l'ombre desquels il se promenait agréablement, goûtant à plaisir les différents fruits que les branches chargées tendaient vers sa main [...]. Les sommets des montagnes qui entouraient complètement l'endroit étaient presque tous couverts d'arbustes en fleurs. Quelques-uns formaient de charmants refuges, où les sources qui jaillissaient fertilisaient les hautes plantes qui y poussaient $[. . .]^{23}$ ». 

culture $^{24}$. D'autre part, ce lieu est caractérisé par sa fermeture, renvoyant aux rêveries de l'intimité ${ }^{25}$. A cette image symbolique se superpose sans aucun doute celle du paradis originel. Mirtilo rencontre en effet, comme nous l'avons signalé précédemment une femme qui se trouve dans l'état de pureté originelle. Cependant, cette vallée merveilleuse, fertile, où Mirtilo va s'installer est aussi intéressante au niveau diégétique

\subsection{Le fonctionnement diégétique de l'espace}

La notion de trajet spatial nous semble ici primordiale et à la base même du programme narratif général de l'œuvre dont le début s'ordonne de la façon suivante :

1. Situation initiale : Mirtilo atteint l'âge adulte

2. Contrat: Quête de l'idéal de vie

3. Compétence : Talent poétique

Avec ce même programme de départ se succèdent trois séquences, correspondant chacune à un espace différent.

Séquence 1 : la ville

4. Réalisation : Impossibilité d'intégration, rencontre d'obstacles

5. Résultat : Échec

6. Situation finale : désir de à la campagne pour devenir berger

Séquence $2:$ la transhumance

4. Réalisation : Intégration au groupe des bergers

5. Résultat: Succès (provisoire)

6. Situation finale : Déséquilibre car Mirtilo n'est toujours pas installé et n'a pas trouvé la femme idéale

Séquence 3 : la vallée

4. Réalisation : Mirtilo trouve le lieu de ses rêves et la femme désirée

5. Résultat : Succès

6. Situation finale : Réalisation probable de l'idéal de vie

L'issue heureuse était annoncée par une prolepse, sous la forme de prophétie faite par le magicien Ortón ${ }^{26}$ :

«Tes intentions sont louables; elles le seront bien plus lorsqu'à l'élevage de tes moutons, tu uniras la culture des champs, dans la demeure qui t'attend, où la Fortune, en récompense de ta sage et ferme résolution, te destine une innocente et belle jeune fille pour épouse ${ }^{27}$ ».

Le fonctionnement diégétique de l'espace renvoie par ailleurs à trois thèmes littéraires : l'échec de Mirtilo en ville permet d'introduire le Menosprecio de carte y alabanza de aldea et d'expliquer la décision du héros de partir à la campagne. Ensuite, le roman fait la synthèse entre les deux "âges d'or ", tels qu'ils sont développés dans l'œuvre de Virgile. D'une part la vallée découverte par Mirtilo rappelle l'âge d'or de l'églogue IV des Bucoliques, c'est-à-dire l'abondance sans travail, d'autre part, la prophétie d'Ortón envisage l'âge d'or sous son aspect agricole, comme dans le livre 
deuxième des Géorgiques ${ }^{28}$. Cependant le fonctionnement de l'œuvre s'explique aussi par des éléments historiques extérieurs.

\section{LE SYMBOLISME IDÉOLOGIQUE}

\subsection{Espace et esthétique romanesque} genres et leurs canons, leurs topoï. Mais il intègre aussi des discours qui au départ n'ont rien de littéraire en les transformant et en les adaptant à sa structure. Le discours économique est sans nul doute le plus présent dans la structure spatiale du Mirtilo.

Dirtilo; il s'insère même de façon contradictoire, remettant en cause ce qui constitue la thématique même de l'œuvre. A plusieurs reprises, ce roman sur les bergers annonce la fin et le remplacement des troupeaux transhumants par des troupeaux sédentaires et l'intensification des labours par la mise en culture des pâturages. Cette idée apparaît d'abord sous forme de prophétie faite par le magicien Ortón, décrivant l'espace futur :

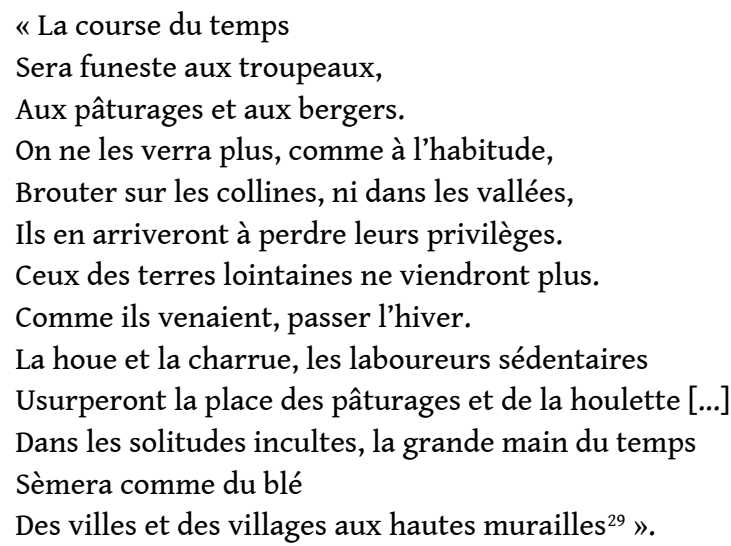


53

\section{conseil :}

«Tes intentions sont louables; elles le seront bien plus lorsqu'à l'élevage de tes moutons, tu uniras la culture des champs, dans la demeure qui t'attend $[. . .]^{30} »$.

Lors de l'arrivée du troupeau aux alentours de Mérida, C'est Mirtilo lui-même qui réfléchit sur l'utilité de la transhumance tout en contemplant l'espace qui l'entoure :

«Comme c'était la première fois que Mirtilo arrivait là. il s'étonnait beaucoup de voir ce riche et fertile territoire, ce Royaume, peuplé seulement de bergers transhumants, car il savait qu'au temps des Romains, c'était une des provinces les plus peuplées et les plus florissantes [...]

Mirtilo discutait de cela avec Silvanio, surpris que depuis tant d'années, on n'ait jamais pensé à remédier le manque de population de ce territoire, capable de faire vivre des milliers d'habitants, qui par leur industrie, leur commerce et leurs arts auraient accru sa richesse naturelle, qui à elle seule serait de loin supérieure à celle que l'Espagne percevait de tous ses troupeaux ; car aussi grande que fût l'utilité des laines et des troupeaux, elle n'équivaudrait jamais à celle des fruits que pourraient donner autant de terrains en friche, utilisés comme pâturages, qui pourraient être peuplés et cultivés [...].

A cela. Mirtilo ajoutait que, ceux qui attribuaient le manque de population de ce Royaume aux troupeaux et aux privilèges des éleveurs qui les amenaient à l'hivernage, étaient dans l'erreur ; car lui jugeait que c'était au contraire l'effet de la désertification même et non sa cause [...].

Il disait aussi que l'Espagne ne perdrait pas ses laines, même si les transhumances cessaient, de la même façon que l'Angleterre, où il n'y avait pas de telles émigrations hivernales, ne les perdait pas $[. . .]^{31}$ »

Les arguments du héros, en faveur de la mise en culture des pâturages ne sont pas sans rappeler ceux du corregidor de Cáceres qui estimait (de façon un peu trop optimiste) que la population de l'Extrémadure pourrait être multipliée par dix, si l'on mettait en culture toutes les terres ${ }^{32}$. Cependant ici, à l'inverse du Memorial ajustado, le dépeuplement n'est pas imputé aux troupeaux et aux privilèges de la Mesta. Enfin, la réflexion sur les avantages des troupeaux sédentaires ressemble fort à une réponse aux sujets posés à la même époque - El Mirtilo a été terminé en 1786 - par les Sociétés Économiques d'Amis du Pays qui se préoccupaient de savoir «Quels sont les moyens de développer solidement l'agriculture d'un pays sans porter préjudice à l'élevage et la façon de surmonter les obstacles qui pourraient s'y opposer ${ }^{33}$ ". Mirtilo essaie en quelque sorte de résoudre la contradiction, mais de manière vague, et en rejetant de toute façon la solution dans le futur. Cependant, le trajet spatial du héros qui abandonne les bergers pour rechercher une vallée où s'installer démontre que la solution choisie tourne le dos à la transhumance et privilégie l'agriculture sédentaire. Symboliquement, ce choix peut aussi signifier un retour aux origines mêmes du roman pastoral. En effet, l'espace pastoral est traditionnellement celui des troupeaux sédentaires.

Le début du roman, où Mirtilo abandonne la ville s'inscrit dans un mouvement de revalorisation de la campagne. A la même époque, on écrit une foule de poésies faisant l'éloge de la vie aux champs, elles aussi en correspondance avec les préoccupations économiques du moment ${ }^{34}$.

Hormis le problème ponctuel posé par la transhumance. El Mirtilo se situe dans un contexte où il s'agissait, de manière plus générale de trouver une solution agricole à l'augmentation de la population. Généralement, les économistes proposèrent des 
solutions qui n'entamaient en rien la structure de la propriété, fortement inégalitaire, et qui freinait en partie l'augmentation de la production. Le roman de Montengón sans suivre vraiment l'idéologie dominante, aboutit inconsciemment sur un aveu d'impuissance. La solution de la vallée vierge trouvée par le héros est une rêverie, un espace idéal, utopique, bien que la prophétie du mage laisse entrevoir le choix d'une agriculture sédentaire. Si Mirtilo s'installe dans l'Eden pour reconstruire, peut-être, un monde meilleur, c'est que le monde existant ne lui offre aucune autre alternative.

\section{CONCLUSION}

L'étude systématique de l'espace constitue une approche fructueuse à plusieurs niveaux. Dans une perspective esthétique, elle fait apparaître les contradictions de la vraisemblance romanesque oscillant entre les canons du roman pastoral et un « réalisme mimétique ».

Ensuite, l'espace s'impose comme une catégorie textuelle à part entière, qui structure le déroulement de l'œuvre. Il n'est pas un élément isolé ou décoratif mais remplit plusieurs fonctions au sein du roman : fonction esthétique, symbolique, narrative. Son analyse permet aussi d'atteindre les implications idéologique de l'œuvre.

Montengón propose un retour à l'état de nature avec l'obligation de cultiver la terre, avatar de l'âge d'or des Géorgiques. L'espace utopique proposé à la fin du roman, tout comme le trajet spatial du héros révèlent l'inconscient collectif d'une société en voie d'urbanisation qui contemple avec nostalgie ses racines campagnardes. Cependant, cette nostalgie de l'espace rural doit aussi être mise en relation avec la littérature économique et le contexte social de l'époque. Dans une conjoncture de prix agricoles à la hausse dû à l'augmentation de la population, les élites urbaines lettrées ont tendance à valoriser la campagne sous toutes ses formes. De plus, la fuite dans l'espace pastoral est peut être aussi une façon d'exorciser et d'oublier les problèmes urbains, nombreux et traumatisants pour cette même classe: forte immigration rurale, délinquance, émeutes de subsistance, troubles sociaux provoqués la plupart du temps par les mauvaises récoltes.

\section{NOTES}

1. Mikhaïl Bakhtine, Esthétique et théorie du roman, Paris, Gallimard, 1978, p. 472.

2. Avalle Arce, Juan Bautista, La novela pastoril en España, Madrid, ed. Istmo, 1974, p. 13 : “[...] después de Los pastores del Betis, de don Gonzalo de Saavedra, (que apareció en 1633). no se volvió más a escribir otra novela de este tipo, al menos que haya llegado a mi conocimiento".

3. Voir Marc Marti, Ville et campagne dans l'Espagne des Lumières (1746-1808), Saint Étienne. 1994. thèse de doctorat nouveau régime, chapitre 8.

4. Avalle Arce, ibid., p. 28.

5. Voir l'introduction critique de Eusebio, par Fernando García Lara, Madrid, Biblioteca del Pensamiento Hispánico, 1984, p. 39. 
6. Voir à ce propos Jacques Soubeyroux, «Le discours du roman sur l'espace, approche méthodologique ». Lieux dits. Recherches sur l'espace dans les textes ibériques. Publications de l'Université de Saint-Étienne, Saint Étienne, 1993, p. 13 à 22.

7. Voir Ernst Curtius, La littérature européenne et le Moyen Age latin, Paris, PUF, 1956, p. 319, dénombre un minimum de trois éléments et un maximum de sept pour le locus amœnus :

8. El Mirtilo o los pastores trashumantes, op. cit., p. 68 : “[...] Montano quiso pararse en un barranco, bastante frondoso, por medio del cual iba serpeando lentamente un manso arroyuelo entre la crecida yerba, donde podía pacer, y abrevarse el ganado a su satisfacción. Ellos se recogieron a la sombra de tres grandes alcornoques, donde no quiso Montano que estuviesen ociosos los rabeles de sus zagales".

9. Toujours situés cependant dans un locus amœnus.

10. Mikhail Bakhtine, op. cit., p. 237-238 définit le chronotope comme «ce qui se traduit littéralement par "temps-espace"; la corrélation essentielle des rapports spatiaux-temporels telle qu'elle a été assimilée par la littérature ».

11. Ernst Curtius, op. cit., p. 319.

12. El Mirtilo, op. cit., p. 58 :

"Con cantos amorosos

Y gorgeos suaves.

Los bosques ya frondosos

Hacen sonar las aves".

13. El Mirtilo [...], op. cit., p. 215 à 217.

14. Littéralement. Mépris de la ville et éloge de la vie aux champs

15. El Mirtilo, ibid.. p. 15 :

“[...] Yo en mi pobreza

Aparente disfruto, porque nada

Me inquieta en tal estado,

$\mathrm{Ni}$ inquietar el alma sosegada

Que el fasto de las ciudades

Prefiere aquestas soledades".

16. El Mirtilo, ibid., p. 280-281 :

"Tendrás, no hay duda alguna,

Aquí entre los pastores

Una dicha más pura ; otros contentos

De las penas exentos,

Que enturbiar suelen siempre los de aquellos

Que los reciben sólo de su suerte,

En excelsos empleos, y riqueza.

Pues dicen que son raras

Las almas que allí quedan satisfechas. [...]

Pueden bien sus cabellos

Perfumar con aromas peregrinos,

$\mathrm{Y}$ arrastrar seda en oro entretejida [...]

No es mayor el consuelo del rico, que del pobre".

17. El Mirtilo, ibid., p. 284 :

"Mi padre enseñarme solía [...]

El horror a las ciudades,

Y al lujo, que allí domina,

Como a todas sus altas vanidades.

Ensalzaba al contrario la dulzura

De la paz en la vida campesina, 
Y su preciosa libertad, exenta

De servil atadura [...]".

18. El Mirtilo, ibid., p. 33 : “Al día siguiente se les proporcionó hacer alto en un valle muy fértil y frondoso, donde podía pacer libremente el ganado, y ellos descansar a la sombra de unos acebuches, junto a una fuente, que se despeñaba con gran murmullo de un vecino cerro. Allí, mientras los dos zagalillos que Montano traía se ocupaban en disponer la comida, rogó Montano a Silviano y a Mirtilo que cantasen una canción".

19. Pour les deux types de vraisemblance (discursive et mimétique) au XVIIIe siècle en Espagne voir les théories de Luzán, la Poética, ed. de 1737 y 1789, Madrid, ed. Cátedra, 1974.

20. Durand, Gilbert, Les structures anthropologiques de l'imaginaire, Paris, Bordas, 1984 (10ème édition), p. 437.

21. Gilbert Durand, ibid. p. 280.

22. El Mirtilo, p. 310 :

“Terreno paraíso.

Cuya nueva hermosura me enagena,

Y el alma me arrebata".

23. El Mirtilo, p. 334 : “Do quiera que Mirtilo volvía los ojos se le presentaban varios bosquecillos. separados en hermoso desorden, formados algunos de ellos de árboles fructíferos, bajo cuya amena sombra caminaba, gustando a su placer los diversos frutos que le ofrecían a la mano sus cargadas copas [...] Las cimas de los cerros que encerraban aquel sitio en toda su extensión, estaban casi todas vestidas de floridos arbustos. Formaban algunos de ellos senos amenísimos, donde las fuentes que allí tenían su manantial, fertilizaban las altas plantas, que habían allí crecido [...]".

24. Voir par exemple l'églogue IV des Bucoliques de Virgile, Pollion.

25. Gilbert Durand, op. cit., p. 280.

26. Nous prendrons ici le terme de prolepse non dans son sens rhétorique de "prévenir les objections en se les faisant soi-même et en les détruisant d'avance » (Littré), mais dans son sens narratif, défini par Gérard Genette, Figures III, Paris, Seuil, 1977, p. 82 : « [Nous désignerons] par prolepse toute manœuvre narrative consistant à raconter ou à évoquer d'avance un événement ultérieur ».

27. El Mirtilo, p. 81 : “Tus intenciones son loables; lo serán mucho más, cuando al cuidado de tus ovejas, unirás el del cultivo del campo, en la morada que se te espera, donde la suerte, en premio de tu sabia y fuerte resolución, te tiene destinada una inocente y linda doncella por esposa".

28. Dans ce deuxième livre, la vie aux champs et les activités agricoles sont semblables à « la vie que menait sur les terres Saturne d'or ».

29. El Mirtilo, op. cit., p. 52-53 :

"La carrera [del tiempo]

Funesta ha de ser a los ganados,

A pastos, y a pastores, de manera.

Que no se verán más por los collados.

Ni por valles pacer, como solían

Llegarán a perder hasta sus fueros.

Ni tampoco vendrán como venían.

Los de tierras lejanas con calderos.

Usurparán el azadón y arado,

Y los establecidos labradores.

El asiento a los pastos y al cayado [...]

Sembrará en las valdías soledades.

La gran mano del tiempo, como trigo.

Torreadas aldeas y ciudades [...]". 
30. El Mirtilo, op. cit., p. 81 : “Tus intenciones son loables ; lo serán mucho más, cuando al cuidado de tus ovejas, unirás el del cultivo del campo, en la morada que se te espera [...]".

31. El Mirtilo, ibid., p. 264 à 266 : "Como era la primera vez que Mirtilo llegaba allí, extrañaba mucho el ver aquel rico y fértil suelo, y Reino, poblado sólo de advenidizos pastores, sabiendo él que era una de las provincias más poblada y floreciente en tiempo de los Romanos [...]

De esto trataba Mirtilo con Silvanio, maravillándose que de tantos años atrás, no se hubiese jamás pensado en remediar la falta de población de aquel suelo capaz de mantener millares de habitadores, que con su industria, comercio y artes, acrecentarían su riqueza natural, que por sí sola sería muchas veces mayor a la que España percibía de todos sus ganados; pues por grande que fuese la utilidad de las lanas, y de las crías, no equivaldría de mucho a sólo los productos que pudieran dar tantos terrenos valdíos, y destinados a los pastos, si en vez de éstos, estuviesen poblados y cultivados [...]

A esto añadía Mirtilo que le parecía padecer engaño aquellos que atribuían la falta de población de aquel Reino a los ganados, y a los fueros de los ganaderos que los conducían a invernar; pues juzgaba que esto era, al contrario, efecto de la despoblación misma, y no causa de ella[...]

Decía también que España no perdería sus lanas, aunque cesasen las trashumaciones de los pastores, como no las perdía Inglaterra, donde no había tales emigraciones invernales [...]"

32. Marc Marti, op. cit.. chapitre 3.

33. "Quáles son los medios de fomentar sólidamente la agricultura en un país sin detrimento de la cría de ganados, y el modo de remover los obstáculos que pueden impedirlo" Le thème revient à plusieurs reprises entre 1777 et 1785, voir notre travail de thèse déjà cité, chapitre 3 et chapitre 6.

34. Voir supra, introduction.

\section{AUTEUR}

\section{MARC MARTI}

Université de Nice-Sophia Antipolis 